\title{
Validação do questionário de qualidade de vida (King's Health Questionnaire) em mulheres brasileiras com incontinência urinária
}

\author{
Validation of a quality of life questionnaire (King's Health Questionnaire) \\ in Brazilian women with urinary incontinence
}

\begin{abstract}
Eliana Suelotto Machado Fonseca ${ }^{1}$, Adriana Luciana Moreno Camargo ${ }^{2}$, Rodrigo de Aquino Castro ${ }^{3}$, Marair Gracio Ferreira Sartori ${ }^{4}$, Marcelo Cunio Machado Fonseca ${ }^{5}$, Geraldo Rodrigues de Lima ${ }^{6}$, Manoel João Batista de Castello Girão
\end{abstract}

\section{RESUM0}

Objetivo: a proposta deste estudo foi traduzir e validar o King's Health Questionnaire (KHQ) para mulheres brasileiras com incontinência urinária. Métodos: 134 pacientes com incontinência urinária, confirmada pelo estudo urodinâmico, foram recrutadas em ambulatório de Uroginecologia. Inicialmente, traduzimos o questionário KHQ para a língua portuguesa (do Brasil) de acordo com critérios internacionais. Devido às diferenças da língua, fizemos a adaptação cultural, estrutural, conceitual e semântica do KHQ, para que as pacientes compreendessem as questões. Todas as pacientes responderam duas vezes o KHQ, no mesmo dia, com dois entrevistadores distintos, com intervalo de 30 minutos de uma entrevista para a outra. Depois de 7 a 14 dias, a aplicação do questionário foi repetida numa segunda visita. Foram testadas a confiabilidade (consistência interna intra e inter-observador) e validade do constructo e discriminativa. Resultados: foram necessárias várias adaptações culturais até obtermos a versão final. A consistência interna intra-observador (alfa de Cronbach) das diversas dimensões oscilou de moderada a alta $(0,77-0,90)$ e a consistência interna inter-observador oscilou de 0,66 a 0,94 . $\mathrm{Na}$ validação do constructo, obtivemos correlação de moderada a forte entre os domínios específicos para incontinência urinária e manifestações clínicas que, sabidamente, afetam a qualidade de vida dessas pacientes. Conclusão: o KHQ foi adaptado ao idioma português e para a cultura brasileira, mostrando grande confiabilidade e validade, devendo ser incluído e utilizado em qualquer estudo brasileiro de incontinência urinária.

PALAVRAS-CHAVE: Incontinência urinária; Qualidade de vida; Questionários; Saúde da mulher; Validade

\section{ABSTRACT}

Purpose: the proposal of the present study was to translate and to validate King's Health Questionnaire (KHQ) for Brazilian women with urinary incontinence. Methods: a hundred and thirty-four patients with urinary incontinence, confirmed by urodynamic study, were enrolled from the outpatient clinic of Uroginecology. Initially, we translated the KHQ into the Brazilian Portuguese language in agreement with international criteria. Due to language and cultural differences we performed a cultural, structural, conceptual, and semantic adaptation of the KHQ, in order to make sure that patients were able to fully understand the questions. All patients answered the KHQ twice on the same day, within an interval of 30 min, applied by two different interviewers. After 7 to 14 days, on a second visit, the questionnaire was applied again. Reliability (intra- and interobserver internal consistency), construct and discriminative validity were tested. Results: several cultural adaptations were necessary until we reached the final version. The intra-observer internal consistency (alpha of Cronbach) of the several dimensions varied from moderate to high (0.77-0.90), and the interobserver internal consistency varied from 0.66 to 0.94. Moderate to strong correlation was detected among the specific KHQ urinary incontinence dominions and clinical

1 Enfermeira Responsável do Ambulatório de Uroginecologia e Mioma da Universidade Federal de São Paulo - Escola Paulista de Medicina (UNIFESP)

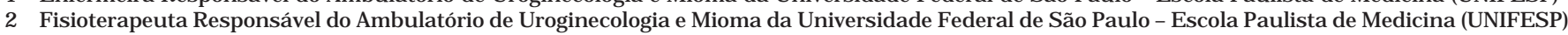

3 Médico Chefe do Ambulatório de Uroginecologia e Mioma da Universidade Federal de São Paulo - Escola Paulista de Medicina (UNIFESP)

4 Professora Adjunta; Chefe do Setor de Uroginecologia do Departamento de Ginecologia (Setor de Uroginecologia) da UNIFESP

5 Médico do Grupo de Pesquisa Clínica em Ginecologia da UNIFESP - Departamento de Ginecologia (Setor de Uroginecologia) da UNIFESP

6 Professor Titular; Departamento de Ginecologia (Setor de Uroginecologia) da UNIFESP

7 Professor Adjunto Livre Docente; Departamento de Ginecologia (Setor de Uroginecologia) da UNIFESP

Correspondência: Eliana Suelotto Machado Fonseca

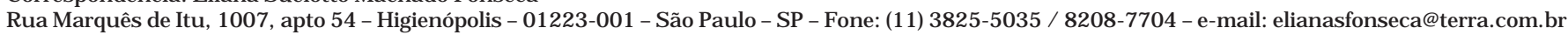
O questionário validado em português poderá ser obtido por solicitação à autora pelo e-mail elianasfonseca@terra.com.br 
urinary incontinence manifestations known to affect the quality of life of these patients. Conclusion: KHQ was adapted to the Portuguese language and to the Brazilian culture, showing great reliability and validity. It should be included and used in any Brazilian urinary incontinence clinical trial.

KEYWORDS: Urinary incontinence; Quality of life; Questionnaires; Women's health; Validity

\section{Introdução}

A perda da continência urinária pode afetar até $50 \%$ das mulheres em alguma fase de suas vidas $^{1}$. A vida social dessas pacientes passa a depender da disponibilidade de banheiros. Elas comumente relatam preocupação e embaraço com o odor de urina. Apresentam dificuldade durante o intercurso sexual, por medo de perder urina ou de precisar interrompê-lo para urinar, além de alterações do sono $^{2,3}$.

Entre 30 e 50\% das pessoas que sofrem de incontinência urinária não relatam espontaneamente esse fato ao médico ou à enfermeira, e só procuram o serviço de saúde após o primeiro ano do início dos sintomas por acharem que a perda de urina é esperada com o evoluir da idade ${ }^{4}$. Elas, silenciosamente, têm redução da auto-estima, tornando-se deprimidas, angustiadas e irritadas.

O envolvimento do próprio paciente no seu tratamento e a necessidade de avaliação mais ampla de como e quanto determinada doença ou intervenção médica atinge a qualidade de vida são fundamentais em qualquer serviço de saúde ${ }^{5}$. A mensuração da qualidade de vida ajuda a selecionar e monitorar problemas psico-sociais de um paciente, demonstra a percepção da população sobre diferentes problemas de saúde e mede os resultados das intervenções médicas ${ }^{5}$. Por isso, a Sociedade Internacional de Continência (ICS) tem recomendado que um questionário de qualidade de vida seja incluído em todo e qualquer estudo sobre incontinência urinária ${ }^{6}$.

O termo e o conceito de qualidade de vida surgiram com o crescimento e o desenvolvimento econômico ocorridos após a Segunda Guerra Mundial, nos Estados Unidos da América, para descrever efeito de aquisição de diferentes bens na vida das pessoas ${ }^{7}$. A evolução tecnológica e os conceitos de economia em saúde tornaram necessária a avaliação não só da eficácia e da toxicidade das intervenções, mas também do seu impacto na qualidade de vida dos indivíduos ${ }^{7,8}$. O conceito de qualidade de vida é subjetivo e, portanto, sua definição é variável. Está relacionado à percepção do indivíduo sobre o seu estado de saúde em grandes domínios ou dimensões de sua vida ${ }^{5,9}$.
O interesse em mensurar a qualidade de vida, em relação aos cuidados de saúde, tem aumentado nos últimos anos, de tal modo que a WHO (World Health Organization) iniciou projeto de avaliação internacional de qualidade de vida, levando em consideração decisões de tratamento, autorização para novos fármacos e política de pesquisa ${ }^{10}$. Os instrumentos de medida de qualidade de vida são, em sua maior parte, desenvolvidos em outras línguas e culturas que não a nossa. Utilizam-se, na mensuração da qualidade de vida, questionários que têm por finalidade avaliar os diversos aspectos e dimensões da vida da paciente, como o físico (mobilidade, autocuidado, exercícios), o psicológico (depressão, ansiedade, preocupação), o social (suporte, contato, atividades nas horas vagas), o desempenho geral (emprego, trabalhos domésticos, compras), a dor, o sono e sintomas específicos da doença, por exemplo, perda urinária ${ }^{6-11}$.

Existem vários questionários para se avaliar a qualidade de vida em mulheres incontinentes que têm semelhanças e diferenças entre si. Podem ser utilizados questionários genéricos ou específicos, que avaliam aspectos próprios da gravidade e do impacto dos sintomas na vida das pacientes $^{12}$. Os genéricos, como o SF-36, são de fácil administração e compreensão, mas têm como inconveniente apresentar dimensões gerais, que podem ser pouco sensiveis às alterações clínicas que as pacientes venham a apresentar.

Já os questionários específicos avaliam aspectos próprios da gravidade e do impacto dos sintomas na vida das pacientes, como: Bristol Female Lower Urinary Tract Symptoms (BFLUTS), Quality of Life in Persons with Urinary Incontinence (I-QOL), Stress Incontinence Questionnaire (SIQ) e King's Health Questionnaire (KHQ).

O questionário Bristol Female Lower Urinary Tract Symptoms é, essencialmente, usado para medir o impacto dos sintomas da incontinência urinária. As questões focam mais o grau de incômodo do que o problema causado aos pacientes ${ }^{12}$.

Já os questionários Quality of Life in Persons with Urinary Incontinence (I-QoL) e Stress Incontinence Questionnaire (SIQ) medem o impacto dos sintomas urinários de maneira geral. As questões são utilizadas para avaliar todos os domínios da qualidade de vida e as frases são construídas para atingir 
somente pacientes com incontinência urinária, evitando co-morbidades ${ }^{12}$.

Dentre os questionários específicos, destaca-se o KHQ por usar ambos os métodos de avaliação, tanto a presença de sintomas de incontinência urinária, quanto seu impacto relativo, o que leva a resultados mais consistentes. Permite mensuração global e também avalia o impacto dos sintomas nos vários aspectos da individualidade na qualidade de vida ${ }^{12}$.

Como a incontinência urinária tem grande impacto na qualidade de vida das pacientes, um instrumento para mensuração da qualidade de vida permitirá avaliar o resultado do tratamento escolhido e facilitará a comparação de estudos realizados em locais diferentes, utilizando tratamentos diferentes, em populações semelhantes ${ }^{13,14}$. Por este motivo, escolhemos o KHQ para ser traduzido e validado em nosso meio.

Cumpre ressaltar que a adaptação cultural das perguntas do questionário é pré-requisito para a correta tradução, considerando-se que é impossivel a transmissão exata de pensamentos e de idéias de uma língua para outra. É, portanto, necessário dar ênfase à tradução conceitual, e não à semântica ${ }^{15}$.

Diante da relevância do tema e à falta de estudos nacionais que avaliaram o impacto da incontinência urinária na qualidade de vida, propusemo-nos a realizar o presente estudo.

\section{Métodos}

O presente estudo refere-se a um trabalho prospectivo a fim de avaliar um questionário de qualidade de vida para pacientes com incontinência urinária (o KHQ), realizado no setor de Uroginecologia e Cirurgia Vaginal da Disciplina de Ginecologia Geral da UNIFESP-EPM. No total, foram incluídas 114 pacientes, das quais 60 participaram da fase de adaptação cultural e 54 da fase de validação do questionário (teste e reteste).

Todas as pacientes se submeteram ao estudo urodinâmico, que confirmou o diagnóstico de incontinência urinária e classificou a incontinência em esforço, mista ou bexiga hiperativa. Não foram incluídas pacientes com doenças degenerativas e/ou neurológicas associadas, para que nenhum outro fator associado pudesse interferir na avaliação da qualidade de vida.

Todas as pacientes foram submetidas ao teste do absorvente, classificando-as em incontinência urinária leve (teste de 0 a 25 gramas), moderada ( 26 a 50 gramas), grave (51 a 75 gramas) e muito grave (76 a 100 gramas).
Inicialmente, foi solicitado autorização à autora do questionário do King's Health, Professora Doutora Linda Cardozo, para a sua utilização. Após o recebimento da autorização, começamos a fase de adaptação cultural com a tradução do questionário de acordo com os critérios internacionais ${ }^{15}$. Dois professores de inglês, independentes, brasileiros e cientes do objetivo da pesquisa, dando ênfase à tradução conceitual, realizaram as chamadas de tradução 1 e 2 do questionário original em inglês ${ }^{16}$. Essas duas traduções foram discutidas pelos dois tradutores e pela coordenadora do estudo, em reunião de consenso, até a obtenção da versão 1 . As discrepâncias encontradas foram discutidas em reunião com a equipe médica do Setor de Uroginecologia e Cirurgia Vaginal do Departamento de Ginecologia da UNIFESP. Assim, originou-se a versão 2.

O KHQ é composto por trinta perguntas que são arranjadas em nove domínios. Relatam, respectivamente, a percepção da saúde, o impacto da incontinência, as limitações do desempenho das tarefas, a limitação física, a limitação social, o relacionamento pessoal, as emoções, o sono e a energia e as medidas de gravidade. Existe também uma escala de sintomas que é composta pelos seguintes itens: freqüência urinária, noctúria, urgência, hiperreflexia vesical, incontinência urinária de esforço, enurese noturna, incontinência no intercurso sexual, infecções urinárias e dor na bexiga. Há, também, um espaço para a paciente relatar qualquer outro problema que ela possa ter relacionado com a bexiga.

A todas as respostas são atribuídos valores numéricos, somados e avaliados por domínio. Os valores são, então, calculados por meio de fórmula matemática, obtendo-se, assim, o escore de qualidade de vida, que varia de 0 a 100 , considerando-se que quanto maior o número obtido, pior a qualidade de vida.

O questionário obtido foi, então, aplicado em 20 pacientes, com idades entre 18 e 82 anos, que apresentavam incontinência urinária e que não haviam se submetido a nenhum tratamento clínico, em período inferior a 15 dias.

Aplicaram-se as 30 perguntas do KHQ da versão 2 , acrescidas da opção "não aplicável”, com a finalidade de identificar questões não compreendidas ou não executadas, que foram consideradas culturalmente impróprias. As questões que tiveram índice de "não aplicável" superior a 15\% por esse grupo de pacientes foram substituídas por outras de mesmo conceito, modificando-se a redação da versão 2 do questionário, criando-se, então, a versão $3^{17}$.

Essa versão foi, então, aplicada a outras 20 pacientes e obtiveram-se, ainda, algumas altera- 
ções conceituais, porém, em menor grau. Todas as questões que ainda tiveram mais de $15 \%$ de "não aplicável" foram modificadas, dando origem à $4^{\mathrm{a}}$ versão. Esta foi aplicada a mais 20 pacientes e, por apresentar índice menor que $15 \%$ de resposta "não aplicável" para todas as questões, tornou-se a versão final (Anexo 1). Com esse questionário já pronto, passamos à fase de validação do KHQ.

Cinqüenta e quatro pacientes foram entrevistadas em dois momentos, no mesmo dia, separadamente, por dois entrevistadores (1 e 2) distintos, e após período médio de 8 dias da primeira entrevista, uma segunda entrevista foi feita pelo entrevistador 1 (autora do estudo). O tempo para aplicação do questionário variou de 5 a 20 minutos.

A validação final do $\mathrm{KHQ}$ foi realizada por meio da avaliação da confiabilidade e validade ${ }^{5}$. Como medidas de confiabilidade foram usadas a consistência interna, o teste reteste e a reprodutibilidade $^{17}$.

A validade do constructo foi determinada demonstrando-se a consistência das mensurações do KHQ com outras medidas do fenômeno da incontinência urinaria, como freqüência urinária, urgência miccional, noctúria e bexiga hiperativa $^{12,17,18}$. A validade discriminante foi demonstrada mostrando-se a variação da qualidade de vida segundo a gravidade da incontinência ${ }^{17}$.

A análise estatística descritiva utilizou freqüência e média de Bussab e Morettin, desvio padrão e mediana (intervalo). Para medida de confiabilidade foram utilizados a consistência interna, por meio do coeficiente de Cronbach padronizado, e o teste reteste, avaliado pelo coeficiente de correlação intraclasses. O coeficiente de Pearson foi empregado para demonstrar a reprodutibilidade, a correlação entre os parâmetros clínicos e os diferentes domínios do KHQ e a validade do constructo ${ }^{17,18}$. Foi utilizado o programa SPSS 10.0 para Windows.
Este estudo foi avaliado e aprovado pelo Comitê de Ética e Pesquisa da Universidade Federal de São Paulo - Hospital São Paulo (UNIFESP-EPM), sendo que todas as pacientes que participaram da pesquisa assinaram termo de consentimento antes de responder ao questionário.

\section{Resultados}

As 54 pacientes incluídas na fase de avaliação tinham média de idade 55 anos e mediana de 53 anos (variando de 34 a 82 anos). A maioria (51,9 $\%)$ era casada ou vivia com parceiros, $94,4 \%$ eram alfabetizadas, 51,9\% não trabalhavam fora e 74,1\% nunca haviam procurado o serviço de saúde para tratar da incontinência. A média de partos foi de 4,0, a de micções diurnas de 6,5 e a de noturnas de 2,9. A média de absorventes empregados por dia foi de 1,5 e o tempo de incontinência era de 5,6 anos.

Todas as pacientes tiveram o diagnóstico confirmado por estudo urodinâmico e a média do teste do absorvente foi de 25,4 g. Da série total, $11,1 \%$ tinham diagnóstico de incontinência urinária mista, 5,5\% de bexiga hiperativa e 83,4\% apresentavam incontinência urinária de esforço.

A Tabela 1 mostra a consistência interna e a correlação intraclasses intra-observador e interobservador.

O valor alfa de Cronbach nas correlações intra e interobservadores foram maiores que 0,82 em todos os domínios do questionário, com exceção do "impacto da incontinência", entre as respostas dadas a cada questão, dentro de cada domínio, para o mesmo entrevistador e também para dois entrevistadores em dois momentos diferentes. Isso demonstra haver forte correlação entre as respostas dadas, comprovando a confiabilidade do questionário.

Tabela 1 - Consistência interna intra e interobservador por meio do $\alpha$ de Cronbach e do coeficiente de correlação intraclasses - CCl

\begin{tabular}{|c|c|c|c|c|}
\hline \multirow[b]{2}{*}{ Domínios } & \multicolumn{2}{|c|}{ Intra-observador } & \multicolumn{2}{|c|}{ Interobservador } \\
\hline & $\alpha$ de Cronbach & $\mathrm{CCl}$ & $\alpha$ de Cronbach & $\mathrm{CCl}$ \\
\hline Percepção da saúde & - & & 0,85 & 0,85 \\
\hline Impacto da incontinência & - & & 0,66 & 0,66 \\
\hline Limitações das atividades diárias & 0,85 & 0,85 & 0,85 & 0,85 \\
\hline Limitação física & 0,84 & 0,84 & 0,90 & 0,90 \\
\hline Limitação social & 0,90 & 0,90 & 0,88 & 0,88 \\
\hline Relacionamento pessoal & 0,87 & 0,87 & 0,88 & 0,88 \\
\hline Emoções & 0,87 & 0,87 & 0,92 & 0,92 \\
\hline Sono/energia & 0,77 & 0,77 & 0,84 & 0,84 \\
\hline Medidas de gravidade & 0,82 & 0,82 & 0,93 & 0,93 \\
\hline
\end{tabular}


Observa-se na Tabela 2 a confiabilidade segundo a reprodutibilidade. Demonstra-se que o entrevistador repete o questionário para a paciente e obtém os mesmos escores. Há reprodução adequada da mensuração nos diversos domínios.

Tabela 2 - Confiabilidade segundo a reprodutibilidade.

\begin{tabular}{lcc}
\hline Domínios & $\begin{array}{c}\text { Coeficiente } \\
\text { de Pearson }\end{array}$ & $\begin{array}{c}\text { Intervalo de } \\
\text { confiança }\end{array}$ \\
\hline Percepção da saúde & $0,36^{*}$ & $0,10-0,56$ \\
Impacto da incontinência & $0,60^{*}$ & $0,39-0,74$ \\
Limitações das atividades diárias & $0,75^{*}$ & $0,60-0,84$ \\
Limitaçãofísica & $0,74^{*}$ & $0,59-0,84$ \\
Limitação social & $0,82^{*}$ & $0,71-0,89$ \\
Relacionamento pessoal & $0,78^{*}$ & $0,61-0,88$ \\
Emoções & $0,76^{*}$ & $0,63-0,86$ \\
Sono/energia & $0,62^{*}$ & $0,43-0,76$ \\
Medidas de gravidade & $0,71^{*}$ & $0,54-0,82$ \\
\hline
\end{tabular}

*Todas as correlações são significativas com $p<0,01$.

Ao analisarmos os parâmetros clínicos da incontinência urinária e os diferentes domínios observamos que a urgência, juntamente com a incontinência urinária de esforço, influenciou as atividades diárias $(0,47$ e 0,41), limitações físicas $(0,49$ e 0,356$)$, limitação social $(0,57$ e 0,32$)$ e sono e disposição $(0,44$ e 0,47$)$. A enurese noturna correlacionou-se com limitação das atividades diárias $(0,32)$ e com o sono e disposição $(0,43)$. A freqüência de micções diurnas e a noctúria revelaram correlação moderada e forte em relação à limitação das atividades diárias $(0,46$ e 0,38), limitações físicas $(0,48$ e 0,53$)$, limitações sociais $(0,53$ e 0,47$)$, relações pessoais $(0,40$ e 0,30$)$, emoções $(0,49$ e 0,26$)$ sono e disposição $(0,492$ e 0,508$)$ e medidas de gravidade $(0,496$ e 0,29), o que denota que quanto maior o número de sintomas relatados pelas pacientes, pior a qualidade de vida (Tabela 3).

A validação do constructo do questionário foi demonstrada com a utilização do teste de correlação de Pearson (Tabela 4). Obtivemos correlação de moderada a forte, entre os domínios específicos para incontinência urinária e as manifestações clínicas que, sabidamente, afetam a qualidade de vida das pacientes com incontinência urinária (freqüência, urgência, noctúria e bexiga hiperativa). Os domínios inespecíficos, percepção da saúde e impacto da incontinência tiveram fraca correlação (Tabela 4).

A validação discriminativa é ilustrada na Figura 1, que mostra de forma clara que a qualidade de vida, avaliada pelo $\mathrm{KHQ}$, variou conforme a maior ou menor gravidade da incontinência urinária. Assim, as pacientes com incontinência urinária leve tiveram menores escores de impacto na qualidade de vida em comparação às afetadas pela incontinência urinária moderada, grave e muito grave, respectivamente. A forma final do questionário é apresentada no Guadro 1.

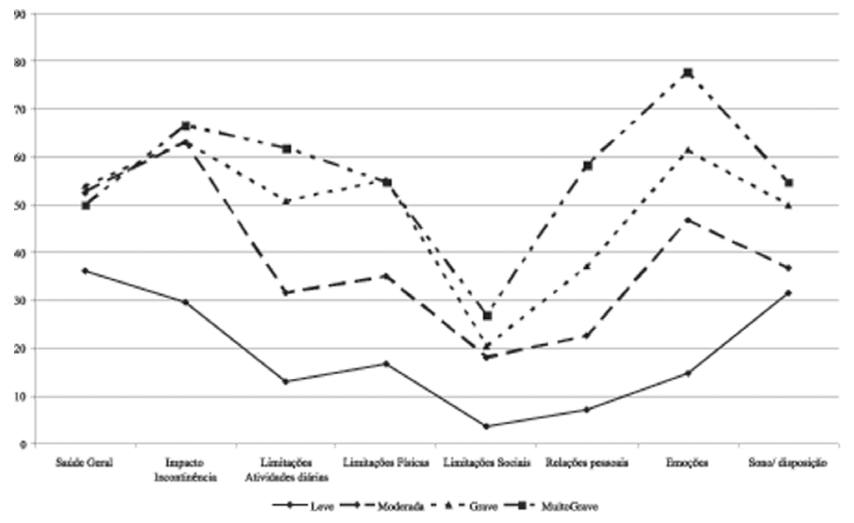

Figura 1 - Pontuação segundo o domínio medidas de gravidade.

Tabela 3 - Correlação de Pearson entre parâmetros clínicos e os domínios do questionário.

\begin{tabular}{|c|c|c|c|c|c|c|c|c|c|c|}
\hline Domínios & 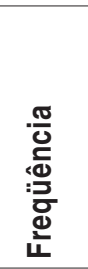 & 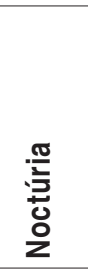 & 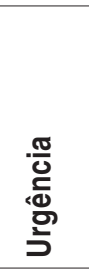 & 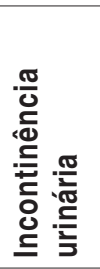 & 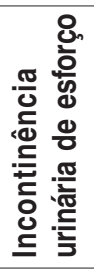 & 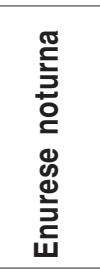 & 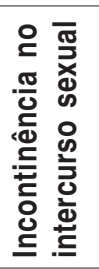 & 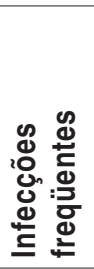 & 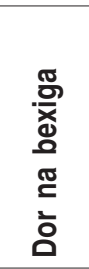 & 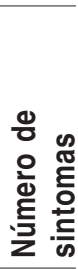 \\
\hline Saúde geral & 0,13 & 0,09 & 0,09 & $-0,04$ & $-0,04$ & 0,23 & 0,07 & 0,16 & $-0,03$ & 0,23 \\
\hline Impacto da incontinência & 0,26 & 0,01 & 0,30 & 0,13 & 0,17 & 0,09 & 0,14 & 0,10 & 0,25 & 0,26 \\
\hline Limitações das atividades diárias & $0,46^{*}$ & $0,38^{*}$ & $0,47^{*}$ & $0,50^{*}$ & $0,41^{*}$ & $0,32^{*}$ & $0,40^{*}$ & $0,31^{*}$ & 0,14 & $0,50^{*}$ \\
\hline Limitações físicas & $0,48^{*}$ & $0,53^{*}$ & $0,49^{*}$ & $0,52^{*}$ & $0,35^{\star}$ & 0,23 & $0,54^{*}$ & $0,42^{*}$ & 0,15 & $0,63^{*}$ \\
\hline Limitações sociais & $0,53^{*}$ & $0,47^{*}$ & $0,57^{*}$ & $0,52^{*}$ & $0,32^{*}$ & 0,24 & $0,49^{*}$ & $0,33^{*}$ & 0,16 & $0,58^{*}$ \\
\hline Relações pessoais & $0,40^{*}$ & $0,30^{*}$ & 0,21 & $0,33^{*}$ & 0,20 & $-0,07$ & $0,70^{*}$ & 0,26 & 0,23 & 0,38 \\
\hline Emoções & $0,49^{*}$ & 0,26 & 0,25 & 0,25 & $0,33^{*}$ & 0,22 & $0,38^{*}$ & $0,27^{\star}$ & 0,09 & $0,48^{*}$ \\
\hline Sono/disposição & $0,49^{*}$ & $0,51^{*}$ & $0,44^{*}$ & $0,52^{*}$ & $0,47^{*}$ & $0,43^{*}$ & $0,51^{*}$ & 0,23 & 0,26 & $0,64^{*}$ \\
\hline Medidas de gravidade & $0,50^{*}$ & $0,29^{*}$ & $0,43^{*}$ & $0,53^{*}$ & $0,42^{*}$ & 0,19 & $0,40^{*}$ & $0,35^{\star}$ & 0,14 & $0,49^{*}$ \\
\hline
\end{tabular}


Tabela 4 - Coeficiente de Pearson para a validade do constructo.

\begin{tabular}{lcccc}
\hline Domínios & Freqüência & Urgência & Noctúria & Bexiga hiperativa \\
\hline Saúde geral & 0,14 & 0,09 & 0,09 & $-0,05$ \\
Impacto da incontinência & 0,26 & 0,31 & 0,02 & 0,13 \\
Limitações das atividades diárias & $0,47^{\star}$ & $0,48^{*}$ & $0,39^{*}$ & $0,50^{*}$ \\
Limitações físicas & $0,48^{*}$ & $0,49^{*}$ & $0,53^{*}$ & $0,53^{\star}$ \\
Limitações sociais & $0,53^{*}$ & $0,58^{*}$ & $0,48^{*}$ & $0,52^{*}$ \\
Relações pessoais & $0,40^{*}$ & 0,22 & $0,30^{*}$ & $0,33^{*}$ \\
Emoções & $0,49^{*}$ & 0,26 & 0,26 & 0,26 \\
Sono/disposição & $0,49^{*}$ & $0,44^{*}$ & $0,51^{*}$ & $0,52^{*}$ \\
Medidas de gravidade & $0,50^{*}$ & $0,43^{*}$ & $0,29^{*}$ & $0,53^{*}$ \\
\hline
\end{tabular}

${ }^{*}$ Todas as correlações são significativas com $p<0,05$.

Quadro 1 - Questionário de qualidade de vida em incontinência urinária após validação.

Nome:__anos
Idade:_ at___

Como você avaliaria sua saúde hoje?

Muito boa ( ) Boa ( ) Normal ( ) Ruim ( ) Muito ruim ( )

Quanto você acha que seu problema de bexiga atrapalha sua vida?

Não ( ) Um pouco ( ) Mais ou menos ( ) Muito ( )

Abaixo estão algumas atividades que podem ser afetadas pelos problemas de bexiga. Quanto seu problema de bexiga afeta você?

Gostaríamos que você respondesse todas as perguntas.

Simplesmente marque com um "X" a alternativa que melhor se aplica a você.

\section{Limitação no desempenho de tarefas}

Com que intensidade seu problema de bexiga atrapalha suas tarefas de casa (ex., limpar, lavar, cozinhar, etc.)

Nenhuma ( ) Um pouco ( ) Mais ou menos ( ) Muito ( )

Com que intensidade seu problema de bexiga atrapalha seu trabalho, ou suas atividades diárias normais fora de casa como: fazer compra, levar filho à escola, etc.?

Nenhuma ( ) Um pouco ( ) Mais ou menos ( ) Muito ( )

\section{Limitação fisica/social}

Seu problema de bexiga atrapalha suas atividades físicas como: fazer caminhada, correr, fazer algum esporte, etc.?

Não ( ) Um pouco ( ) Mais ou menos ( ) Muito ( )

Seu problema de bexiga atrapalha quando você quer fazer uma viagem?

Não ( ) Um pouco ( ) Mais ou menos ( ) Muito ( )

Seu problema de bexiga atrapalha quando você vai a igreja, reunião, festa?

Não ( ) Um pouco ( ) Mais ou menos ( ) Muito ( )

Você deixa de visitar seus amigos por causa do problema de bexiga?

Não ( ) Um pouco ( ) Mais ou menos ( ) Muito ( )

\section{Relações pessoais}

Seu problema de bexiga atrapalha sua vida sexual?

Não se aplica ( ) Não ( ) Um pouco ( ) Mais ou menos ( ) Muito ( )

Seu problema de bexiga atrapalha sua vida com seu companheiro?

Não se aplica ( ) Não ( ) Um pouco ( ) Mais ou menos ( ) Muito ( )

Seu problema de bexiga incomoda seus familiares?

Não se aplica ( ) Não ( ) Um pouco ( ) Mais ou menos ( ) Muito ( )

Gostaríamos de saber quais são os seus problemas de bexiga e quanto eles afetam você. Escolha da lista abaixo APENAS AQUELES PROBLEMAS que você tem no momento.

Quanto eles afetam você?
Freqüência: Você vai muitas vezes ao banheiro?

Um pouco ( ) Mais ou menos ( ) Muito ( )

Noctúria: Você levanta a noite para urinar?

Um pouco ( ) Mais ou menos ( ) Muito ( )

Urgência: Você tem vontade forte de urinar e muito difícil de controlar?

Um pouco ( ) Mais ou menos ( ) Muito ( )

Bexiga hiperativa: Você perde urina quando você tem muita vontade de urinar?

Um pouco ( ) Mais ou menos ( ) Muito ( )

Incontinência urinária de esforco: Você perde urina com atividades físicas como: tossir, espirrar, correr?

Um pouco ( ) Mais ou menos ( ) Muito ( )

Enurese noturna: Você molha a cama à noite?

Um pouco ( ) Mais ou menos ( ) Muito ( )

Incontinência no intercurso sexual: Você perde urina durante a relação sexual?

Um pouco ( ) Mais ou menos ( ) Muito ( )

Infecções freqüentes: Você tem muitas infecções urinárias?

Um pouco ( ) Mais ou menos ( ) Muito ( )

Dor na bexiga: Você tem dor na bexiga?

Um pouco ( ) Mais ou menos ( ) Muito ( )

Outros: Você tem algum outro problema relacionado a sua bexiga?

Um pouco ( ) Mais ou menos ( ) Muito ( )

Emoções

Você fica deprimida com seu problema de bexiga?

Não ( ) Um pouco ( ) Mais ou Menos ( ) Muito ( )

Você fica ansiosa ou nervosa com seu problema de bexiga?

Não ( ) Um pouco ( ) Mais ou Menos ( ) Muito ( )

Você fica mal com você mesma por causa do seu problema de bexiga?

Não ( ) Às vezes ( ) Várias vezes ( ) Sempre ( )

Sono/Energia

Seu problema de bexiga atrapalha seu sono?

Não ( ) Às vezes ( ) Várias vezes ( ) Sempre ( )

Você se sente desgastada ou cansada?

Não ( ) Às vezes ( ) Várias vezes ( ) Sempre ( )

Algumas situações abaixo acontecem com você? Se tiver o quanto?

Vocêusaalgum tipo de protetorhigiênico como: fralda, forro, absorvente tipo Modess paramanter-se seca?

Não ( ) Às vezes ( ) Várias vezes ( ) Sempre ( )

Você controla a quantidade de líquido que bebe?

Não ( ) Às vezes ( ) Várias vezes ( ) Sempre ( )

Você precisa trocar sua roupa íntima (calcinha), quando fica molhadas?

Não ( ) Às vezes ( ) Várias vezes ( ) Sempre ( )

Você se preocupa em estar cheirando urina?

Não ( ) Às vezes ( ) Várias vezes ( ) Sempre ( )

\section{Discussão}

Houve muita dificuldade em fazer a adaptação cultural, pois, embora a maioria de nossas pacientes não fosse analfabeta, foram necessárias várias alterações de palavras para que compreendessem as questões. Logo no início surgiram as primeiras dificuldades, como a necessidade de substituir a palavra "razoavelmente" por "mais ou menos". Havia questões de difícil entendimento, pois simplesmente elas não compreendiam o signifi- 
cado de alguns termos como, por exemplo, "vida social". Foi preciso, então, elaborar a questão esclarecendo-a por meio de exemplos próprios do diaa dia para obtermos respostas condizentes. Como era sabido que o problema estava na interpretação das perguntas, colocamos alguns exemplos, como foi o caso do "protetor higiênico", que substituímos por "absorvente". Ainda assim, tivemos que modificá-lo, porque para as pacientes entrevistadas a palavra absorvente, por várias vezes, não foi compreendida (39\%). Esse problema foi sanado quando acrescentamos absorvente "tipo Modess ${ }^{\circledR}$ ", que por ser marca consagrada e do conhecimento de praticamente todas as mulheres, levou à compreensão da questão.

Isso decorre do fato de que a norma culta, a escrita, usada pela elite e pelos intelectuais para impor às pessoas o modo certo de falar, é bem diferente da norma falada, própria das classes menos intelectualizadas e que tiveram pouca oportunidade de se dedicar aos estudos. Daí, ao se depararem com a escrita, há dificuldade em entendê-la. Defrontamos com a tradução "palavra por palavra" procurando manter o sentido do texto, pois tratando-se de trabalho científico as palavras deviam ser exatas, claras e objetivas.

Assim, apesar de originalmente elaborado para o autopreenchimento pela paciente, optamos pela aplicação com entrevistas para evitarmos viés metodológico caso misturássemos autopreenchimento com entrevistas. Além disso, podemos citar o trabalho de Weinberger et al. ${ }^{19}$, que detectaram que mais de dois terços dos pacientes preferiam que o questionário SF-36 fosse aplicado por entrevistas.

A confiabilidade mensurada pela consistência interna por meio do coeficiente á de Cronbach variou de 0,66 a 0,93 nos dominios impacto da incontinência e medidas de gravidade, respectivamente. Estes intervalos são extremamente semelhantes aos encontrados por Badia Llach et al. ${ }^{18}$ na validação do KHQ para o espanhol. A variação por eles encontrada foi de 0,65 para medidas de gravidade e 0,92 para relações pessoais. Já na versão inglesa ${ }^{6}$, original, o coeficiente á de Cronbach variou de 0,72 a 0,89 para limitação física e relações pessoais, respectivamente, o que também é compativel com os valores por nós encontrados. Sintomas que sabidamente se correlacionam com incontinência urinária, como freqüência, urgência, noctúria e bexiga hiperati$\mathrm{va}^{6,17}$, também se correlacionam significativamente com os domínios específicos ligados à incontinência urinária. Os domínios gerais, percepção da saúde e impacto da incontinência se correlacionaram fracamente com estes sintomas, o que era de se esperar, pela dimensão menos específica em relação à incontinência urinária que estes domínios apresentam.
O instrumento também se mostrou capaz de distinguir os diferentes graus de incontinência urinária, apresentando uma pior qualidade de vida quanto maior a gravidade da incontinência urinária. Novamente, como era de se esperar, nos domínios gerais esta discriminação não se demonstrou.

Por meio do questionário também se pôde mostrar quais os fatores que afetam a qualidade de vida das pacientes com incontinência urinária. Assim, o número de micções diurnas e noturnas referidas foi o fator que mais prejudicou a qualidade de vida. $\mathrm{O}$ aumento da freqüência urinária se relacionou com a maior limitação das atividades da vida diária, levando a alteração dos sentimentos, do relacionamento pessoal e ao isolamento social. Da mesma forma, o número de micções noturnas demonstrou alterar o sono, a energia e as emoções das mulheres incontinentes. Esses achados foram também observados em estudos anteriores ${ }^{18-20}$.

Embora durante a entrevista se tenha notado que a qualidade de vida era pior para as pacientes incontinentes que trabalhavam fora e nas mais jovens, não foi encontrada significância em nossa pesquisa.

Pelo grande impacto da incontinência urinária na vida das pacientes, o profissional de saúde deve almejar e comprometer-se com a melhora da auto-estima e a resolução do seu isolamento social, orientando-a no seu restabelecimento.

Devemos preferir questionários específicos para a condição em estudo, pois com a avaliação específica da qualidade de vida, o profissional conseguirá mensurar o impacto da incontinência urinária. Este instrumento deverá ser utilizado para avaliar a qualidade de vida nas pesquisas clínicas brasileiras envolvendo incontinência urinária, pois permite mensurar o resultado da intervenção terapêutica em estudo, em relação à qualidade de vida da paciente. Além disso, os questionários de qualidade de vida oferecem excelente modo de se iniciar o diálogo entre a paciente e o seu médico, enfermeira ou fisioterapeuta.

Adicionalmente, ainda sob o ponto de vista da qualidade de vida, poderemos comparar os resultados de outros tratamentos em diferentes estudos, desde que, as populações sejam semelhantes e utilizando o KHQ como avaliador principal.

O presente estudo permitiu-nos concluir que o KHQ foi adequadamente traduzido e adaptado para a língua portuguesa do Brasil (Anexo 1) e apresenta alta confiabilidade e validade, devendo ser incluído e utilizado em qualquer estudo brasileiro de incontinência urinária e, se possível, na prática clínica. 


\section{Referências}

1. Luft J, Vriheas-Nichols AA. Identifying the risk factors for developing incontinence: can we modify individual risk? Geriatr Nurs. 1998;19(2):66-70.

2. Coyne KS, Zhou Z, Bhattacharyya SK, Thompson $\mathrm{CL}$, Dhawan R, Versi E. The prevalence of nocturia and its effect on health-related quality of life and sleep in a community sample in the USA. BJU Int. 2003;92(9):948-54.

3. Kizilkaya Beji N, Yalcin O, Ayyildiz EH, Kayir A. Effect of urinary leakage on sexual function during sexual intercourse. Urol Int. 2005;74(3):250-5.

4. Dugan E, Cohen SJ, Bland DR, Preisser JS, Davis CC, Suggs PK, McGann P. The association of depressive symptoms and urinary incontinence among older adults. J Am Geriatr Soc. 2000;48(4):4136.

5. Fitzpatrick R, Fletcher A, Gore S, Jones D, Spiegelhalter D, Cox D. Quality of life measures in health care. In: Applications and issues in assessment. BMJ. 1992;305(6861):1074-7.

6. Kelleher CJ, Cardozo LD, Khullar V, Salvatore S. A new questionnaire to assess the quality of life of urinary incontinent women. Br J Obstet Gynaecol. 1997;104(12):1374-9.

7. Carr AJ, Thompson PW, Kirwan JR. Quality of life measures. Br J Rheumatol. 1996;35(3):275-81.

8. Wyman JF. Quality of life of older adults with urinary incontinence. J Am Geriatr Soc. 1998;46(6):778-9.

9. Ferraz MB. Qualidade de vida. Conceito e um breve histórico. Rev Jovem Med. 1998;3(4):219-22.

10. The World Health Organization Quality of Life assessment (WHOQOL): position paper from the World Health Organization. Soc Sci Med. 1995;41(10):1403-9.

11. DuBeau CE, Kiely DK, Resnick NM. Quality of life impact of urge incontinence in older persons: a new measure and conceptual structure. J Am Geriatr Soc. 1999;47(8):989-94.
12. Kelleher C. Quality of life and urinary incontinence. Baillieres Best Pract Res Clin Obstet Gynaecol. 2000; 14(2):363-79.

13. Hunskaar S, Vinsnes A. The quality of life in women with urinary incontinence as measured by the sickness impact profile. J Am Geriatr Soc. 1991;39(4):378-82.

14. Grimby A, Milsom I, Molander U, Wiklund I, Ekelund $P$. The influence of urinary incontinence on the quality of life of elderly women. Age Ageing. 1993;22(2):82-9.

15. Guillemin F, Bombardier C, Beaton D. Cross-cultural adaptation of health-related quality of life measures: literature review and proposed guidelines. J Clin Epidemiol. 1993;46(12):1417-32.

16. Ciconelli RM, Ferraz MB, Santos W, Meinão I, Quaresma MR. Tradução para a língua portuguesa e validação do questionário de qualidade de vida SF-36 (Brasil SF -36). Rev Bras Reumatol. 1999;39(3): 143-50.

17. Fletcher RH, Fletcher SW, Wagner EH. Clinical epidemiology: the essentials. 3rd ed. Baltimore: Williams \& Wilkins; 1996. p. 19-42.

18. Badia Llach X, Castro Diaz D, Conejero Sugranes J. Validity of the King's Health questionnaire in the assessment of quality of life of patients with urinary incontinence. The King's Group. Med Clin (Barc). 2000; 114(17):647-52.

19. Weinberger M, Oddone EZ, Samsa GP, Landsman PB. Are health-related quality-of-life measures affected by the mode of administration? J Clin Epidemiol. 1996;49(2):135-40.

20. Wagner TH, Patrick DL, Bavendam TG, Martin ML, Buesching DP. Quality of life of persons with urinary incontinence: development of a new measure. Urology. 1996;47(1):67-71. 